\title{
Saliva DNA quality and genotyping efficiency in a predominantly elderly population
}

Harini V. Gudiseva ${ }^{1}$, Mark Hansen², Linda Gutierrez ${ }^{2}$, David W. Collins ${ }^{1}$, Jie He ${ }^{1}$, Lana D. Verkuil ${ }^{1}$, lan D. Danford ${ }^{1}$, Anna Sagaser ${ }^{1}$, Anita S. Bowman ${ }^{1}$, Rebecca Salowe ${ }^{1}$ Prithvi S. Sankar ${ }^{1}$, Eydie Miller-Ellis ${ }^{1}$, Amanda Lehman ${ }^{1}$ and Joan M. O'Brien ${ }^{1 *}$

\begin{abstract}
Background: The question of whether DNA obtained from saliva is an acceptable alternative to DNA from blood is a topic of considerable interest for large genetics studies. We compared the yields, quality and performance of DNAs from saliva and blood from a mostly elderly study population.

Methods: Two thousand nine hundred ten DNAs from primarily elderly subjects (mean age \pm standard deviation (SD): $65 \pm 12$ years), collected for the Primary Open-Angle African-American Glaucoma Genetics (POAAGG) study, were evaluated by fluorometry and/or spectroscopy. These included 566 DNAs from blood and 2344 from saliva. Subsets of these were evaluated by Sanger sequencing $(n=1555)$, and by microarray SNP genotyping $(n=94)$ on an Illumina OmniExpress bead chip platform.

Results: The mean age of subjects was 65 , and $68 \%$ were female in both the blood and saliva groups. The mean \pm SD of DNA yield per $\mathrm{ml}$ of requested specimen was significantly higher for saliva $(17.6 \pm 17.8 \mu \mathrm{g} / \mathrm{ml})$ than blood $(13.2 \pm 8.5 \mu \mathrm{g} / \mathrm{ml})$, but the mean \pm SD of total DNA yield obtained per saliva specimen $(35 \pm 36 \mu \mathrm{g}$ from $2 \mathrm{ml}$ maximum specimen volume) was approximately three-fold lower than from blood (106 $\pm 68 \mu \mathrm{g}$ from $8 \mathrm{ml}$ maximum specimen volume). The average genotyping call rates were $>99 \%$ for 43 of 44 saliva DNAs and $>99 \%$ for 50 of 50 for blood DNAs. For 22 of 23 paired blood and saliva samples from the same individuals, the average genotyping concordance rate was $99.996 \%$. High quality PCR Sanger sequencing was obtained from $\geq 98 \%$ of blood $(n=297)$ and saliva $(n=1258)$ DNAs. DNA concentrations $\geq 10 \mathrm{ng} / \mu \mathrm{l}$, corresponding to total yields $\geq 2 \mu \mathrm{g}$, were obtained for $94 \%$ of the saliva specimens $(n=2344)$.
\end{abstract}

Conclusions: In spite of inferior purity, the performance of saliva DNAs for microarray genotyping was excellent. Our results agree with other studies concluding that saliva collection is a viable alternative to blood. The potential to boost study enrollments and reduce subject discomfort is not necessarily offset by a reduction in genotyping efficiency. Saliva DNAs performed comparably to blood DNAs for PCR Sanger sequencing.

Keywords: Saliva, Blood, DNA, Genotyping, Microarrays, GWAS, SNPs, Elderly, Glaucoma, African-Americans, 260/280 absorbance, Call rates

\footnotetext{
* Correspondence: Joan.O'Brien@uphs.upenn.edu

${ }^{1}$ Scheie Eye Institute, University of Pennsylvania, 51 N. 39th Street,

Philadelphia, PA 19104, USA

Full list of author information is available at the end of the article
} 


\section{Background}

Large epidemiological studies with thousands of participants are increasingly supplementing survey data with genomic DNA [1]. These studies require a simple, noninvasive method of sample collection that yields genomic DNA of adequate quality and quantity for highthroughput technologies [2]. Blood has traditionally been the primary source of genomic DNA, but saliva collection has recently emerged as a viable alternative [2, 3]. In addition to being less invasive, saliva collection has a lower overall cost, lower risk of infection, and simpler logistics. Stabilized saliva specimens can be stored at ambient temperatures for months, whereas blood must be frozen for long term storage, and protected from freeze/thaw cycles [2, 4]. Unlike blood collection, saliva collection does not require a trained phlebotomist; subjects need only to be provided simple directions, and may even donate saliva specimens by mail. These characteristics facilitate community outreach efforts by reducing costs for personnel training and effort, and eliminate the logistical complexities associated with transporting highly perishable blood specimens from remote locations. Saliva collection also leads to significantly higher response rates $[1,5]$, with one study finding a $72 \%$ response rate for saliva collection versus $31 \%$ for blood draws [1]. Another advantage is that saliva DNA has the potential to provide information about the oral microbiome. Despite these advantages, there is still reluctance among the scientific community to use saliva samples, which largely stems from concerns over reduced yield and quality of DNA [6-12].

Most studies agree that oral specimens yield lower quantities of DNA compared to blood [2, 4, 13, 14]. DNA isolated from saliva samples is often contaminated by foreign DNA from bacteria, fungi, and food remnants $[2,13,15]$. Non-human DNA content from saliva samples varies greatly among patients, with studies reporting non-human DNA yields ranging from 23 to $63 \%$ of total DNA [16]. Despite concerns over low yield and variability among samples, previous studies have found that saliva collection still provides sufficient DNA for genotyping $[1,2,4,15,17]$.

Research on the usability of saliva samples from older age groups remains limited, however. Subject age determines, in part, the number of epithelial cells found in saliva $[18,19]$. A previous study found a strong positive correlation between subject age and DNA concentration from saliva samples, with children under age 12 having the lowest DNA concentration [18]. It is important to extend this research to older populations, as the elderly may have veins that are difficult to access for blood collection [2]. Additionally, saliva collection has been shown to reduce anxiety and increase participation rates in older participants [2]. Negative correlation between blood DNA yield and subject age was reported in a large prospective study by Caboux et al by assessing EPIC records of 50,000 subject DNA yields isolated from blood, $14 \%$ of whom were $\geq 65$ [20]. However, saliva collection in the elderly requires further investigation, as hyposalivation can interfere with specimen collection in this age group. Dry mouth has an incidence rate of $30 \%$ in individuals over age 65 [21].

The Primary Open-Angle African-American Glaucoma Genetics (POAAGG) study cohort is the largest AfricanAfrican primary-open angle glaucoma cohort recruited at a single institution (University of Pennsylvania, Department of Ophthalmology, Scheie Eye Institute) to date [22]. The growing size of the cohort $(n=5300)$, older age of POAAGG subjects, improvements in saliva stabilization technologies, and reductions in the amount of DNA needed for next-generation sequencing and genotyping applications have led us to consider saliva collection as the primary method for future subjects. The objective of this study is to examine how well DNA isolated from saliva samples performs, compared to DNA from blood, for array-based genotyping and sequencing. These results will inform the future method of DNA collection for the POAAGG study, as well as other large-scale studies requiring genomic DNA from older populations.

\section{Methods}

\section{Subject recruitment and specimen collection}

The POAAGG study is a five-year population-based project funded by the National Eye Institute of the National Institutes of Health. The study population consists of self-identified Blacks (African Americans, African descent, or African Caribbean). Although subjects as young as age 35 are potentially eligible, primary open-angle glaucoma is typically a disease of old age. Accordingly, enrollment efforts for controls have also preferentially targeted an older population, and the mean age of POAAAGG subjects is approximately 65 . POAAGG subjects were recruited from the University of Pennsylvania from the Scheie Eye Institute, The Perelman Center for Advanced Medicine, and the Mercy Fitzgerald Hospital Ophthalmology satellite. All subjects provided informed written consent, in accordance with the tenets of the Declaration of Helsinki, under University of Pennsylvania IRB-approved protocol 815033.

Blood was collected by venipuncture in $10 \mathrm{ml}$ purple top tubes with EDTA anticoagulant. The maximum volume of blood collected was $8 \mathrm{ml}$ per tube, but sometimes less was obtained. These samples were frozen at $-20^{\circ}$ prior to DNA isolation. For saliva collection, subjects were asked to refrain from drinking or eating $30 \mathrm{~min}$ prior to donating specimens and to remove lipstick. Subjects experiencing dry mouth or difficulties with salivation were 
directed to massage their cheeks with a gentle circular motion to stimulate the salivary glands. Subjects struggling with hyposalivation were also offered packets of sugar, or a sugar substitute, and told to place a small amount on their tongues to induce salivation. A maximum volume of $2 \mathrm{ml}$ of saliva per subject was collected in Oragene DISCOVER (OGR-500) self-collection kits (DNA Genotek, Canada), because we found that delivering more than one $2 \mathrm{ml}$ saliva specimen in a single sitting was challenging for subjects, but most subjects were able to deliver this volume within a few minutes. The saliva specimens were mixed with stabilizing reagent within the collection tubes per manufacturer's instructions, and these were stored at room temperature until DNA extraction.

\section{DNA extraction}

DNA was isolated from freshly thawed blood samples using Gentra PureGene kits (Qiagen, Valencia, CA), and the optional RNase treatment step was included. DNA from saliva samples was extracted using the prepIT.L2P reagent (cat \# PT-L2P-5, DNA Genotek, Canada) and precipitated with ethanol according to manufacturer's instructions. The saliva DNA samples were RNAse treated by double digestion with RNase A and RNase T and re-precipitated using ethanol according to manufacturer's instructions.

\section{DNA quantitation and sample selection}

The concentrations of DNA from blood and saliva samples were determined using the fluorescence-based Quant iT dsDNA Board-Range (BR) assay kit (cat \# Q33130, Life Technologies, CA). Fluorescence was measured with a Tecan Infinite M 200 Pro multimode microplate reader (Tecan, NC). Two thousand nine hundred ten DNAs (566 from blood and 2344 from saliva) from the POAAGG cohort were used to evaluate DNA yields. During November 2014 the POAAGG study switched to from blood to saliva as the primary means of specimen collection, and all available saliva DNAs obtained since then were included. Blood DNAs which had been quantified using Nanodrop spectrophotometry were excluded from analysis to control for potential bias from different quantitation methods. DNA quantification by UV spectrometry may be confounded by RNA or other contamination, and may systematically overestimate DNA concentration. Accordingly, only the blood and saliva samples that had been quantified by the same automated fluorometry protocol were used to compare DNA yields. A subset of this group, 94 DNA samples (50 from blood and 44 from saliva), were selected for microarray analysis. These included 23 pairs of samples from which blood and saliva were obtained from the same individual. The selection of the group of 94 samples was deliberately weighted to include those having unusually high and low DNA concentrations, along with some saliva DNAs that were unusually turbid or discolored. UV absorption spectra from 220 to $340 \mathrm{~nm}$ and 260/280 and 260/230 absorbance ratios were also obtained for this group, using a Nanodrop ND-8000 spectrophotometer (Thermo Scientific, DE), and protein contamination was measured directly with the Qubit protein assay kit (cat \# Q33211, Life Technologies, CA) with a Qubit 2.0 Fluorometer. The 1555 DNA samples chosen for sequencing comprised consecutive samples from early February 2013 to late October 2015, spanning the time interval during which the POAAGG study shifted from blood to saliva collection.

\section{Microarray genotyping and PCR Sanger sequencing}

Ninety-four DNA samples were genotyped in two separate batches using the HumanOmniExpress 24v1 bead chip assay (Illumina, CA) on the Infinium platform by Illumina FastTrack Services (Illumina, San Diego, CA). The genotype calls were generated using the GenomeStudio genotyping module (GT). Cluster optimization, reproducibility analysis for paired samples, and data evaluation were also performed as per standard practices at Illumina FastTrack services. During cluster optimization 1822 markers were removed from 716,503 total markers on the array.

Saliva DNAs were used as templates for PCR targeting glaucoma-associated SNPs for two genes, TMCO1 and $C D K N 2 B-A S 1$. PCR was done in a reaction volume of $12 \mu \mathrm{l}$ using Platinum Taq hot start DNA polymerase (\#106566-034, ThermoFisher.com), dNTP mix (\# 18427088, ThermoFisher.com) and betaine (Sigma \#B0300, http://www.sigmaaldrich.com). Each PCR reaction contained $1.2 \mu \mathrm{l}$ of $10 \mathrm{x}$ Platinum Taq reaction buffer, $0.24 \mu \mathrm{l}$ of $10 \mathrm{mM}$ dNTPs, $0.96 \mu \mathrm{l}$ of $50 \mathrm{mM} \mathrm{MgCl}{ }_{2}(4 \mathrm{mM}$ final), $2.4 \mu \mathrm{l}$ of forward and reverse primer $(2 \mathrm{pmol} / \mu \mathrm{l})$, $3 \mu \mathrm{l}$ saliva DNA, $0.096 \mu \mathrm{l}$ Taq polymerase, $3.6 \mu \mathrm{l} 5 \mathrm{M}$ betaine, and $0.5 \mu \mathrm{l}$ nuclease-free water. For TMCO1, 1555 DNA samples were tested, using forward primer ACCACAGGGAGCCTCTCGTT and reverse primer GCCCTGCCTGCTTTTTAGGGA. For CDKN2B-AS1, the same 1555 samples were tested, using forward primer GCGGAGAAGAATGTCCCGGC and reverse primer GCCAGGAAGGACGAGTCCCC. Thermal cycling was performed on an ABI 9700 instrument using a touchdown protocol: initial denaturation $95 \mathrm{deg} \mathrm{C}$ $5 \mathrm{~min}$; 14 cycles: $94 \mathrm{deg} 20 \mathrm{~s}, 63 \mathrm{deg}$ to $56 \mathrm{deg}$ with 0.5 deg decrement per cycle $20 \mathrm{~s}, 72$ deg 45 s; 25 cycles: $94 \operatorname{deg} 20$ s, $56 \operatorname{deg} 20$ s, $72 \operatorname{deg} 45$ s; $72 \operatorname{deg} 10 \mathrm{~min}$; final hold at $10 \mathrm{deg}$. PCR products were cleaned up by digestion with shrimp alkaline phosphatase (SAP) and exonuclease 1 (Exo1). Cycle sequencing reactions were robotically assembled using a Biomek 3000 automated liquid handling system, with the BigDye Terminator v3.1 
kit (\#4337455, ThermoFisher.com). Cycle sequencing was done in a volume of $5 \mu \mathrm{l}$, containing $1.25 \mu \mathrm{l}$ of a 4fold or 8-fold dilution of the SAP/Exo1 digested PCR product, $1.25 \mu \mathrm{l}$ sequencing primer (one of the PCR primers) at $5 \mathrm{pmol} / \mu \mathrm{l}$, and $2.5 \mu \mathrm{l}$ diluted BigDye v3.1 Ready Mix. Sequencing reactions were cleaned up with BigDye XTerminator kits (\#4376485, ThermoFisher.com). Capillary electrophoresis was done on an ABI 3130xl genetic analyzer with $50 \mathrm{~cm}$ capillary array and POP-7 polymer (\#4363785, ThemoFisher.com), and sequencing chromatograms were aligned, trimmed and scored using Sequencher 5.1 software (GeneCodes Corp, genecodes.com).

\section{Statistical analysis}

Kernel density plots were created using the ggplot2 package in the $\mathrm{R}$ statistical package [23]. Comparisons between blood samples and saliva samples were made using t-tests for comparison of means and chi-squared tests for comparison of proportions. For the comparison of 23 paired samples with blood and saliva from the same subjects, a paired t-test was used. An F-test was used to test for equality of variance between two groups. All these statistical comparisons were made using SAS v9.3 (SAS Institute Inc., Cary, NC), and two-sided $p<0.05$ was considered to be statistically significant. Sequencing results from blood vs. saliva DNAs were compared using a two-sided, two-sample proportion test with STATA v14.1 (StataCorp, College Station, TX).

\section{Results and discussion}

\section{Quality and yield of DNA from saliva}

The saliva samples from our predominately elderly study population yielded DNAs that were often highly viscous, with the majority having noticeable turbidity. Although subjects had been asked to not eat or drink 30 min prior to specimen donation, with the exception of placing a small amount of sugar or sugar substitute on the tongue to stimulate salivation when needed, visible contaminants such as food particles, lipstick, food coloring, tobacco, etc. were sometimes present in the saliva samples. In some cases, brownish discoloration, suspected to be tobacco-related, or reddish contamination (chewing gum, candy, or lipstick) carried through processing and were still visible in some of the purified DNA samples (Fig. 1).

Two thousand three hundred forty-four saliva specimens and 566 blood specimens were obtained, with both blood and saliva specimen obtained from 23 people for purposes of this study, and for a small number of individuals for whom the initial DNA extraction from blood was not successful. The demographics of the two groups of participants were very similar, with mean age 65 years and approximately $68 \%$ female (Table 1 ). The mean

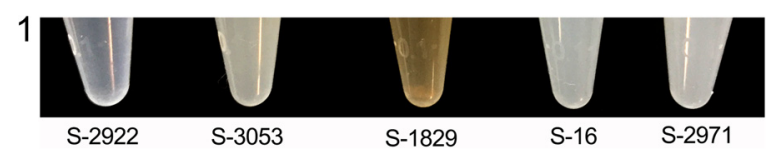

Fig. 1 Examples of saliva DNAs having visible impurities. Sample S-2922 has a normal clear appearance, similar to DNAs extracted from blood. The others have various degrees of turbidity and/or brownish/reddish discoloration. Brownish saliva DNAs, S-3053 and S-1829, may have come from specimens contaminated by tobacco, food dyes or lipstick

$( \pm \mathrm{SD})$ total yield of DNA from the 2344 saliva specimens was $35 \pm 36 \mu \mathrm{g}$, as compared to the $106 \pm 68 \mu \mathrm{g}$ in the 566 blood specimens. However, after accounting for the smaller specimen collection volume that was attempted for saliva $(2 \mathrm{ml})$ vs. blood $(8 \mathrm{ml})$, the mean $( \pm \mathrm{SD})$ yield of DNA per ml saliva specimen was $17.6 \pm 17.8 \mu \mathrm{g} / \mathrm{ml}$, which was significantly higher than that for blood specimens $(13.2 \pm 8.5 \mathrm{ug} / \mathrm{ml}, p<0.0001)$. The higher yield of DNA per $\mathrm{ml}$ of saliva is necessarily offset by the presence of non-human DNA, which, as mentioned above, has been addressed by other studies. A failure rate of $6.0 \%$ (141 subjects from 2344 total) was observed for saliva specimens, with failure defined as a final DNA concentration that was below $10 \mathrm{ng} / \mu \mathrm{l}$ of DNA, which corresponded to less than $2 \mu \mathrm{g}$ yield in the minimum elution volume $(200 \mu \mathrm{l})$. There was a weak negative correlation (Spearman correlation coefficient $r=-0.1$, $p<0.0001$ ) between subject age and DNA yield from saliva samples whereas, the DNA yield from blood was not correlated with age $(r=0.04, p=0.30)$.

The total DNA yield distribution from the larger subset of the POAAGG cohort (566 blood DNAs and 2344 saliva DNAs) was evaluated by the Quant iT assay, and is illustrated as a kernel density plot in Fig. 2. The majority of saliva samples fall in the lower yield region of the plot, as expected, whereas the yield from blood tubes varies widely, with considerable overlap with the yield from saliva, in spite of the 4-fold larger maximum

Table 1 Demographic characteristics and DNA yields per specimen, corresponding to 2910 DNAs included in this study

\begin{tabular}{llll}
\hline & $\begin{array}{l}\text { Blood } \\
(8 \mathrm{ml} \mathrm{max})\end{array}$ & $\begin{array}{l}\text { Saliva } \\
(2 \mathrm{ml} \text { max })\end{array}$ & P-value \\
\hline No. samples & 566 & 2344 & \\
Age \pm SD years & $65 \pm 12$ & $65 \pm 12$ & 0.95 \\
$\begin{array}{l}\text { Female (\%) } \\
\text { Mean } \pm \text { SD for total DNA }\end{array}$ & 67.8 & 67.4 & 0.88 \\
$\begin{array}{l}\text { yield per specimen }(\mu \mathrm{g}) \\
\begin{array}{l}\text { Mean } \pm \text { SD for DNA yield } \\
\text { per ml of requested }\end{array}\end{array}$ & $13.2 \pm 8.5$ & $35 \pm 36$ & $P<0.0001$ \\
specimen $(\mu \mathrm{g} / \mathrm{ml})$ & & $17.6 \pm 17.8$ & $P<0.0001$ \\
\hline
\end{tabular}

Specimen collection volume was up to $8 \mathrm{ml}$ for blood and up to $2 \mathrm{ml}$ for saliva 


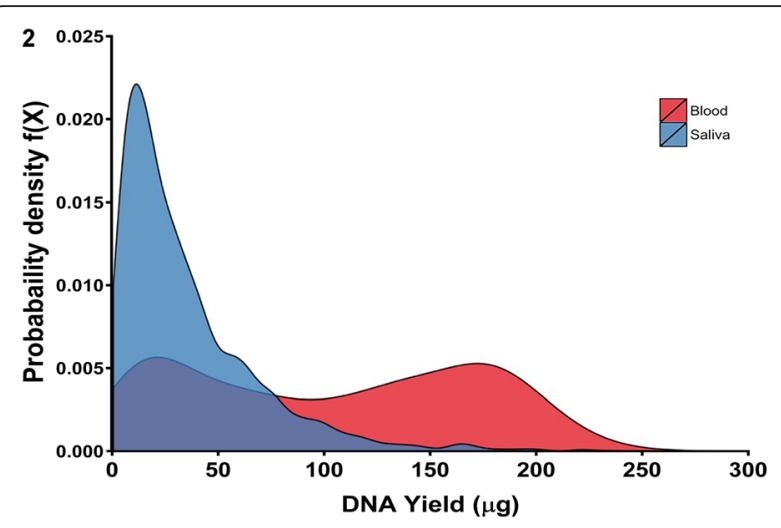

Fig. 2 Density plot summarizing 2910 total DNA yields that were obtained from single blood ( $n=566,8 \mathrm{ml}$ max) and saliva $(n=2344$, $2 \mathrm{ml}$ max) collection tubes

specimen volume $(8 \mathrm{ml}$ vs. $2 \mathrm{ml})$ that was attempted for blood. The distribution of DNA from saliva is relatively narrow with a single peak, whereas the blood DNA distribution is broad and bimodal. Although exact specimen collection volumes were not recorded, we believe this difference is because we often were unable to collect a full $8 \mathrm{ml}$ blood specimen for a substantial fraction of our mostly elderly study population, whereas almost all subjects succeeded in supplying the $2 \mathrm{ml}$ saliva specimen volume that was requested.

Among the 94 samples that were selected for microarray genotyping, average UV absorbance for the 44 saliva DNA samples was higher than for the 50 blood DNA samples across the range from $230 \mathrm{~nm}$ to $340 \mathrm{~nm}$ (Fig. 3a). The higher absorbance at $\mathrm{A}_{230} \mathrm{~nm}$ may be due to the presence of relatively large amounts of carbohydrates and heavily glycosylated mucin in the saliva samples, or sugar that had been given to ameliorate dry mouth. The mean $\mathrm{A}_{260 / 280}$ ratio for saliva DNAs (1.71) was significantly lower than for blood (1.91) $(p<.0001$, Table 2, Fig. 3b). Furthermore, the $A_{260 / 280}$ ratios for saliva DNAs were much more variable than for blood DNAs $(p<0.0001$ for test of equal variance, Table 2, Fig. 3b). The 94 DNA concentrations and quality data, as measured by fluorescence and spectrophotometry are shown in Additional file 1: Table S1, and summarized in Table 2. It is important to note that the mean DNA concentration of the 44 saliva DNAs chosen for genotyping, $78.4 \mathrm{ng} / \mu \mathrm{l}$, was more than 2 -fold lower than for blood $(175.3 \mathrm{ng} / \mu \mathrm{l})(p<0.0001$, Table 2$)$. A minimum concentration of $50 \mathrm{ng} / \mu \mathrm{l}$ is recommended for genotyping with the OmniExpress array, so this study deliberately included many saliva DNAs having sub-optimal concentrations for this purpose.

The turbidity of many DNAs obtained from saliva (Fig. 1), together with the decreased 260/280 absorbance

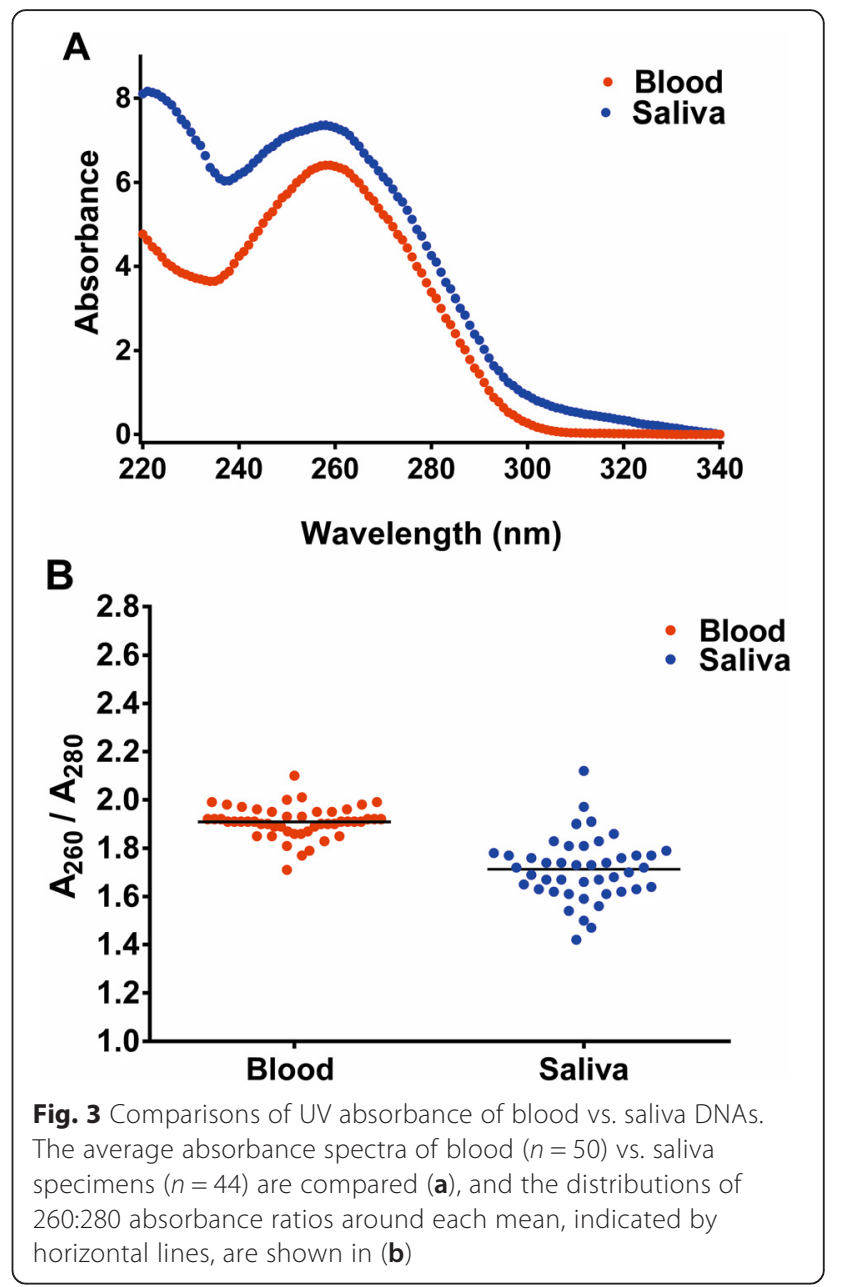

ratio for saliva DNA during spectrophotometry, prompted us to evaluate protein contamination in the 94 DNAs that had been selected for microarray genotyping. Using the Qubit Protein assay, the protein concentrations was below the level of detection in 42 (84\%) of blood samples and in 16 (36\%) of saliva samples $(P<$ 0.0001 , Additional file 1: Table S1). However, among those with protein above the detectable level, we observed no significant difference in the mean protein estimation from blood $(116 \pm 54 \mathrm{ng} / \mu \mathrm{l})$ versus saliva DNA $(119 \pm 59 \mathrm{ng} / \mu \mathrm{l})$.

Table 2 Characteristics of the 94 blood and saliva DNA samples selected for genotyping

\begin{tabular}{llll}
\hline Sample source & $\begin{array}{l}\text { Mean DNA } \\
\text { conc }(\mathrm{ng} / \mu \mathrm{l})\end{array}$ & $\begin{array}{l}\text { Mean } \pm \text { SD } \\
260 / 280\end{array}$ & $\begin{array}{l}\text { Mean } \pm \text { SD } \\
260 / 230\end{array}$ \\
\hline Blood $(n=50)$ & $175.3 \pm 163$ & $1.91 \pm 0.06$ & $1.50 \pm 0.57$ \\
Saliva $(n=44)$ & $78.4 \pm 91.5$ & $1.71 \pm 0.13$ & $1.00 \pm 0.30$ \\
$P$-value & 0.0006 & $P<0.0001$ & $P<0.0001$ \\
\hline
\end{tabular}




\section{Genotyping efficiency, accuracy and PCR Sanger sequencing}

Surprisingly, the average genotyping call rates from the Illumina HumanOmniExpress 24v1 bead chip assay for blood and saliva DNAs were nearly identical: 99.62 vs $99.61 \%$ from blood and saliva, respectively. The individual call rates for the 94 samples, along with age, gender, concentrations and comments on physical appearance of the DNA in solution after extraction are listed in Additional file 1: Table S1. The majority (59\%) of these subjects were age 65 or older. The genotyping call rates are plotted for the two-genotyping batches, with source tissues indicated, in Fig. 4a. If $98 \%$ is considered the minimum threshold for success, then only one outlying sample, S-781 from saliva, failed; the paired blood DNA collected from the same individual yielded a typical passing call rate $(99.5 \%)$. With the exception of this one sample, all saliva DNAs yielded call rates $>99 \%$, whereas the $2^{\text {nd }}$ and $3^{\text {rd }}$ worst performing samples in this survey were blood DNAs (Fig. 4a). The genotype call rates were slightly lower in batch 2 , due to optimized clustering for batch 1.
The relationship between genotyping call rate and DNA concentration of the samples is plotted in Fig. $4 \mathrm{~b}$. We observed genotyping call rates above $99 \%$ on 92 of 94 samples, although the concentration of many was below $10 \mathrm{ng} / \mu \mathrm{l}$, less than $20 \%$ of the minimum (50 ng/ $\mu \mathrm{l})$ recommended for this assay. All low concentration blood DNAs $(<25 \mathrm{ng} / \mu \mathrm{l})$ yielded call rates $99 \%$ or higher, whereas a low concentration $(10.5 \mathrm{ng} / \mu \mathrm{l})$ saliva DNA, S-781 had the lowest call rate (97\%) (Additional file 1: Table S1). The two worst performing blood samples, B-1898 and B-636 with call rates of $98.9 \%$ and $99.0 \%$, had intermediate DNA concentrations $238 \mathrm{ng} / \mu \mathrm{l}$ and $296 \mathrm{ng} / \mu \mathrm{l}$, suggesting no discernable effect of DNA concentration on the call rates for DNA from blood throughout the tested range of $\sim 10$ to $\sim 400 \mathrm{ng} / \mu \mathrm{l}$. Although all but one saliva DNA yielded call rates $>99 \%$, the worst performing saliva samples were clustered at the lowest end of the concentration range (Fig. 4b). However, the average call rate $(99.68 \%)$ for blood DNAs, having concentrations $\leq 25 \mathrm{ng} / \mu \mathrm{l}$, was only slightly higher than for dilute saliva DNAs only $(99.45 \%)$, and this difference is not significant $(p=0.08)$.
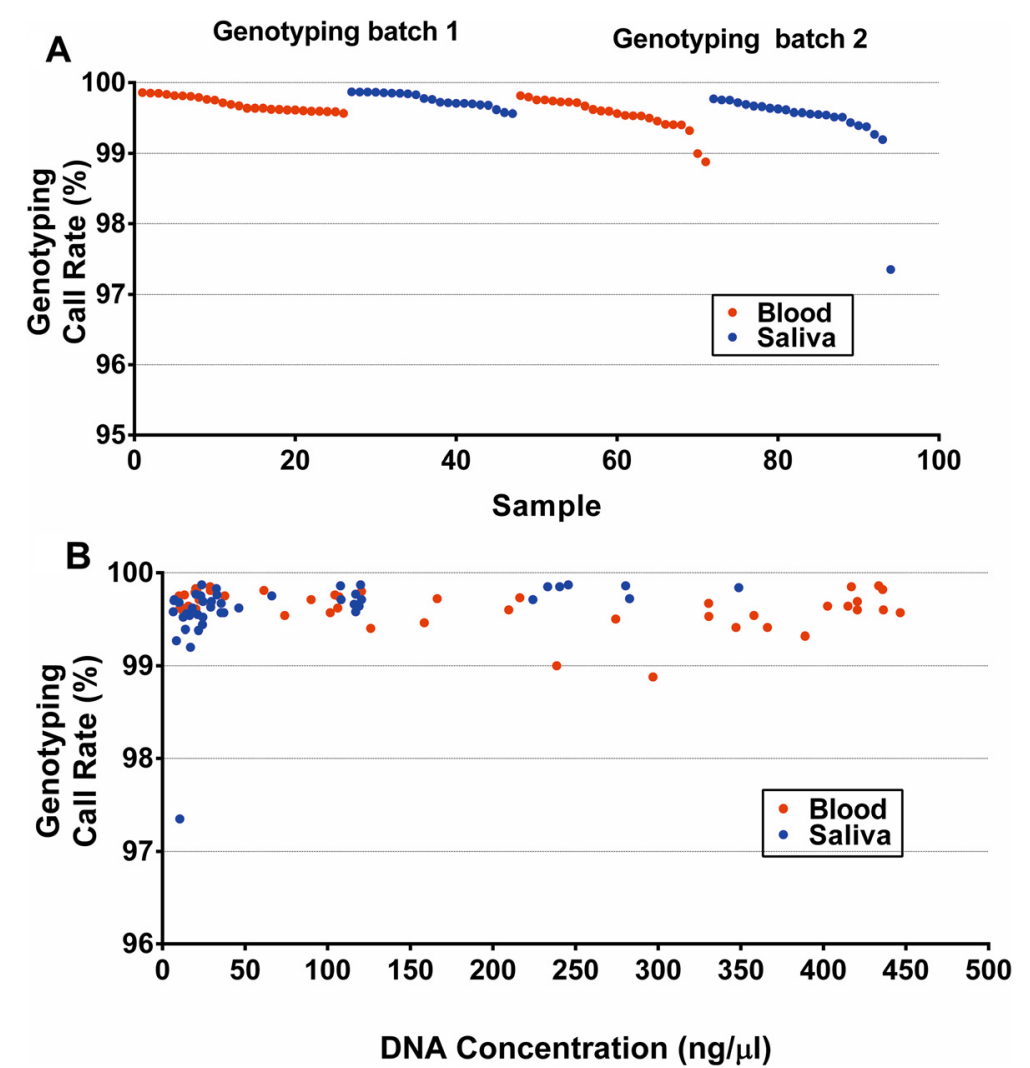

Fig. 4 Comparison of genotyping call rates by Illumina Human OmniExpress microarrays. 94 DNAs from blood or saliva were genotyped in 2 batches $(\mathbf{a})$, and call rates are plotted versus DNA concentration $(\mathbf{b})$. The first batch of samples was deliberately enriched for DNAs having visible turbidity and/or abnormally low or high concentrations. The second batch contained 23 paired specimens: both blood and saliva collected from the same individuals 


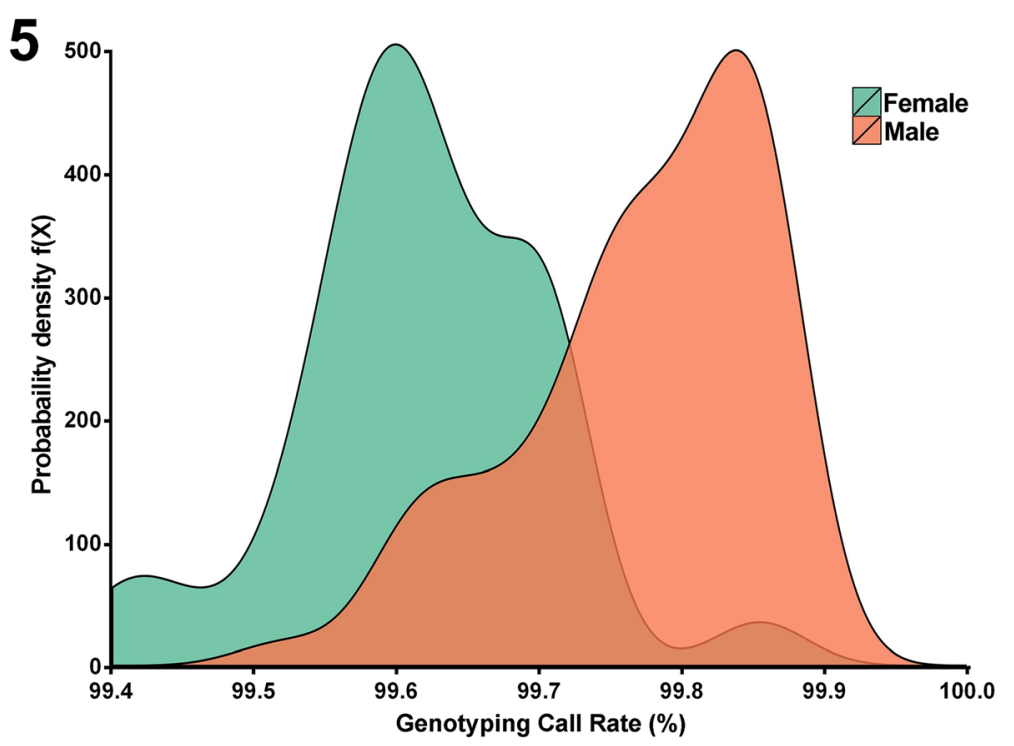

Fig. 5 Comparison of genotyping call rates on the Illumina Human OmniExpress microarrays for male $(n=46)$ vs. female $(n=48)$ subjects

For more concentrated DNAs, > $25 \mathrm{ng} / \mu \mathrm{l}$, the average call rate for saliva DNAs (99.73\%) was actually higher than blood DNAs (99.6\%) ( $p=0.003)$. In general, our results are consistent with those of Bahlo et al. [17] who concluded that genotyping with an Illumina platform was generally robust for saliva DNAs, even though these contain visible impurities and bacterial or other nonhuman DNA.

Genotyping call rates were expected to be slightly higher for males than females, due to the presence of $\mathrm{Y}$ chromosome markers on the array. The difference in the distributions between the genotyping call rates of males vs females is noticeable on the kernel density plot in Fig. 5, although this difference is small in absolute terms. The mean genotyping call rates were $99.5 \%$ for females and $99.7 \%$ for males.

Among 22 paired blood and saliva DNAs isolated from the same individuals, their call rate agreed very well, with a mean difference of $0.083 \%(p=0.46$, paired ttest). One pair of samples was removed from this genotyping concordance estimate on account of the extreme outlier with low genotyping call rate of $97.35 \%$, saliva sample (S-781), discussed above.

One thousand five hundred fifty-five of the saliva DNAs were evaluated by PCR Sanger sequencing, targeting glaucoma-associated SNPs in or near the CDKN2BAS1 and TMCO1 genes. High quality Sanger sequencing (Quality Value $(\mathrm{QV})>25$, KB Basecaller) was obtained for $\geq 98.0 \%$ of these samples for CDKN2B-AS1 and TMCO1 amplicons, from both blood $(n=297)$ and saliva $(n=1258)$ DNAs, and these success rates did not differ significantly (data not shown).

\section{Conclusion}

Although DNAs extracted from saliva were inferior to those from blood in terms of physical appearance and standard measures of quality, their performance in array-based genotyping was excellent, and nearly indistinguishable from DNAs from blood. With the exception of a single saliva DNA sample (1 of 44) having a low call rate of $97.35 \%$, saliva-derived DNA samples yielded call rates $>99 \%$, with genotyping results that were highly concordant with blood DNA from the same subjects. The mean concordance of genotyping calls from the paired saliva-blood samples was at least $99.996 \%$ (Additional file 1: Table S1). Saliva specimens yielded a minimum of $2 \mu \mathrm{g}$ DNA at concentrations above $10 \mathrm{ng} / \mu \mathrm{l}$ for $94 \%$ of specimens ( $n=2344$ extractions). High quality PCR Sanger sequencing data was obtained for $\geq 98 \%$ of blood and saliva DNAs in two independent high throughput sequencing experiments ( $n=1555$ tested). Collection of saliva DNA has the potential to boost study enrollments, thereby increasing the statistical power of large population based studies such as the POAAGG project, while decreasing the personnel effort and training required to obtain DNA samples of adequate quality for microarray-based genotyping and sequencing.

\section{Additional file}

Additional file 1: Table S1. Subject gender, age, DNA characteristics and genotyping results corresponding to the samples used for genotyping. ("B" sample IDs indicate DNA from blood and "S" sample IDs are DNA from saliva). (DOCX $31 \mathrm{~kb}$ ) 


\section{Competing interests}

$\mathrm{MH}$ and LG are currently employed by Illumina, Inc., whose products and services were used in this study. MH, LG and DC have disclosed that they own stock in Illumina. All other authors declared that they have no competing interests.

\section{Authors' contributions}

PS, EE, AL and JO recruited subjects to the study and provided basic demographics of the subjects. JO, HG, and DC designed the study. $\mathrm{HG}, \mathrm{JH}$ and AS extracted DNA from saliva and blood specimens and performed quality analysis. DC, ID, LV performed the PCR Sanger sequencing analysis. $\mathrm{MH}$ and $\mathrm{LG}$ supervised and performed the genotyping. $\mathrm{HG}, \mathrm{DC}, \mathrm{AB}, \mathrm{LV}, \mathrm{ID}$, $\mathrm{MH}$ and $\mathrm{LG}$ analyzed the data. $\mathrm{HG}, \mathrm{DC}$ and $\mathrm{RS}$ drafted the manuscript. All authors have read and approved the manuscript.

\section{Acknowledgments}

Research reported in this publication was supported by the National Eye Institute of the National Institutes of Health under Award Number R01EY023557. Funds also come from the F.M. Kirby Foundation, Research to Prevent Blindness, Macula Vision Research Foundation, The Paul and Evanina Bell Mackall Foundation Trust, and the National Eye Institute, National Institutes of Health, Department of Health and Human Services, under eyeGENE ${ }^{\text {TM }}$ and contract Nos. HHSN260220700001C and HHSN263201200001C. The sponsor or funding organization had no role in the design or conduct of this research. We are grateful to Illumina Inc (San Diego, CA) for their donation of genotyping services and data analysis, Gui-shuang Ying for statistical analysis and critical review of the manuscript, Venkata R M Chavali for helpful discussions, and our Clinical Research Coordinators Laura O' Keefe, Nish Patel, Melissa Homsher, Aaishah Raquib, Rebecca Margolis and Katelyn Gordon for enrolling the study subjects.

\section{Author details}

${ }^{1}$ Scheie Eye Institute, University of Pennsylvania, 51 N. 39th Street,

Philadelphia, PA 19104, USA. ${ }^{2}$ Illumina Inc, San Diego, CA, USA.

\section{Received: 29 July 2015 Accepted: 24 February 2016}

Published online: 07 April 2016

\section{References}

1. Hansen TV, Simonsen MK, Nielsen FC, Hundrup YA. Collection of blood, saliva, and buccal cell samples in a pilot study on the danish nurse cohort: Comparison of the response rate and quality of genomic DNA. Cancer Epidemiol Biomarkers Prev. 2007;16(10):2072-6.

2. Sun F, Reichenberger EJ. Saliva as a source of genomic DNA for genetic studies: Review of current methods and applications. Oral Health Dent Manag. 2014;13(2):217-22.

3. Rylander-Rudqvist T, Hakansson N, Tybring G, Wolk A. Quality and quantity of saliva DNA obtained from the self-administrated oragene method-a pilot study on the cohort of Swedish men. Cancer Epidemiol Biomarkers Prev. 2006;15(9):1742-5.

4. Abraham JE, Maranian MJ, Spiteri I, et al. Saliva samples are a viable alternative to blood samples as a source of DNA for high throughput genotyping. BMC Med Genomics. 2012;5:19-8794-5-19.

5. Peplies J, Fraterman A, Scott R, Russo P, Bammann K. Quality management for the collection of biological samples in multicentre studies. Eur J Epidemiol. 2010;25(9):607-17

6. Freeman B, Powell J, Ball D, Hill L, Craig I, Plomin R. DNA by mail: An inexpensive and noninvasive method for collecting DNA samples from widely dispersed populations. Behav Genet. 1997;27(3):251-7.

7. Hayney MS, Dimanlig P, Lipsky JJ, Poland GA. Utility of a "swish and spit" technique for the collection of buccal cells for TAP haplotype determination. Mayo Clin Proc. 1995;70(10):951-4.

8. Le Marchand L, Lum-Jones A, Saltzman B, Visaya V, Nomura AM, Kolonel LN. Feasibility of collecting buccal cell DNA by mail in a cohort study. Cancer Epidemiol Biomarkers Prev. 2001;10(6):701-3.

9. Garcia-Closas M, Egan KM, Abruzzo J, et al. Collection of genomic DNA from adults in epidemiological studies by buccal cytobrush and mouthwash. Cancer Epidemiol Biomarkers Prev. 2001;10(6):687-96.

10. Richards B, Skoletsky J, Shuber AP, et al. Multiplex PCR amplification from the CFTR gene using DNA prepared from buccal brushes/swabs. Hum Mol Genet. 1993;2(2):159-63.
11. Walker AH, Najarian D, White DL, Jaffe JF, Kanetsky PA, Rebbeck TR. Collection of genomic DNA by buccal swabs for polymerase chain reactionbased biomarker assays. Environ Health Perspect. 1999;107(7):517-20.

12. Walsh DJ, Corey AC, Cotton RW, et al. Isolation of deoxyribonucleic acid (DNA) from saliva and forensic science samples containing saliva. J Forensic Sci. 1992;37(2):387-95.

13. Nunes AP, Oliveira IO, Santos BR, et al. Quality of DNA extracted from saliva samples collected with the oragene DNA self-collection kit. BMC Med Res Methodol. 2012;12:65-2288-12-65.

14. Hu Y, Ehli EA, Nelson K, et al. Genotyping performance between saliva and blood-derived genomic DNAs on the DMET array: A comparison. PLoS ONE. 2012;7(3):e33968.

15. Durdiakova J, Kamodyova N, Ostatnikova D, Vlkova B, Celec P. Comparison of different collection procedures and two methods for DNA isolation from saliva. Clin Chem Lab Med. 2012:50(4):643-7.

16. Nishita DM, Jack LM, McElroy M, et al. Clinical trial participant characteristics and saliva and DNA metrics. BMC Med Res Methodol. 2009:9:71-2288-9-71.

17. Bahlo M, Stankovich J, Danoy P, et al. Saliva-derived DNA performs well in large-scale, high-density single-nucleotide polymorphism microarray studies. Cancer Epidemiol Biomarkers Prev. 2010;19(3):794-8.

18. Gasso P, Pagerols M, Flamarique I, et al. The effect of age on DNA concentration from whole saliva: Implications for the standard isolation method. Am J Hum Biol. 2014;26(6):859-62.

19. El-Mogy M, Simkin M, Haj-Ahmad Y. Comparative study of DNA isolated from saliva prese rved in norgen's preservative using norgen's saliva DNA isolation kit versus qiagen's QIAamp DNA blood mini kit. Norgen Biotek Corporation. 2012 (Application Note 56).

20. Caboux E, Lallemand C, Ferro G, et al. Sources of pre-analytical variations in yield of DNA extracted from blood samples: Analysis of 50,000 DNA samples in EPIC. PLOS ONE. 2012;7(7):e39821.

21. Ship JA, Pillemer SR, Baum BJ. Xerostomia and the geriatric patient. J Am Geriatr Soc. 2002;50(3):535-43.

22. Charlson ES, Sankar PS, Miller-Ellis E, et al. The primary open-angle African American glaucoma genetics study: baseline demographics. Ophthalmology. 2015:122(4):711-20.

23. Wickham H. Ggplot2: Elegant graphics for data analysis. 1st ed. New York: Springer; 2009. Corr. 3rd printing 2010 edition ed.

\section{Submit your next manuscript to BioMed Central and we will help you at every step:}

- We accept pre-submission inquiries

- Our selector tool helps you to find the most relevant journal

- We provide round the clock customer support

- Convenient online submission

- Thorough peer review

- Inclusion in PubMed and all major indexing services

- Maximum visibility for your research

Submit your manuscript at www.biomedcentral.com/submit
) Biomed Central 